\title{
On the Solutions of Difference Equation Systems with Padovan Numbers
}

\author{
Yasin Yazlik $^{1}$, D. Turgut Tollu ${ }^{2}$, Necati Taskara ${ }^{3}$ \\ ${ }^{1}$ Department of Mathematics, Faculty of Science and Art, Nevsehir University, Nevsehir, Turkey \\ ${ }^{2}$ Department of Mathematics-Computer Sciences, Science Faculty, Necmettin Erbakan University, Konya, Turkey \\ ${ }^{3}$ Department of Mathematics, Science Faculty, Selcuk University, Konya, Turkey \\ Email: yyazlik@nevsehir.edu.tr,dttollu@konya.edu.tr,ntaskara@selcuk.edu.tr
}

Received November 1, 2013; revised December 1, 2013; accepted December 8, 2013

Copyright (C) 2013 Yasin Yazlik et al. This is an open access article distributed under the Creative Commons Attribution License, which permits unrestricted use, distribution, and reproduction in any medium, provided the original work is properly cited. In accordance of the Creative Commons Attribution License all Copyrights (C) 2013 are reserved for SCIRP and the owner of the intellectual property Yasin Yazlik et al. All Copyright (C) 2013 are guarded by law and by SCIRP as a guardian.

\section{ABSTRACT}

In this study, we investigate the form of the solutions of the following rational difference equation systems $x_{n+1}=\frac{x_{n-1} \pm 1}{y_{n} x_{n-1}}, \quad y_{n+1}=\frac{y_{n-1} \pm 1}{x_{n} y_{n-1}}$, such that their solutions are associated with Padovan numbers.

Keywords: Rational Difference Equation System; Padovan Numbers; Plastic Number

\section{Introduction}

Nonlinear difference equations have long interested researchers in the field of mathematics as well as in other sciences. They play a key role in many applications such as the natural model of a discrete process. There are many recent investigations and interest in the field of nonlinear difference equations from several authors [1-15]. For example, Tollu et al. [14] investigated the solutions of two special types of Riccati difference equations

$$
x_{n+1}=\frac{1}{1+x_{n}} \text { and } y_{n+1}=\frac{1}{-1+y_{n}}
$$

such that their solutions are associated with Fibonacci numbers. In [2], Aloqeili investigated the stability properties and semi-cycle behavior of the solutions and the form of solutions of the difference equation

$$
x_{n+1}=\frac{x_{n-1}}{a-x_{n} x_{n-1}} .
$$

In [4], author obtained the formulae of solutions of the difference equations

$$
x_{n+1}=\frac{x_{n-2}}{ \pm 1+x_{n} x_{n-1} x_{n-2}} .
$$

Also, he studied the global asymptotic stability of the equilibrium points of these equations via the formulae. In [5], Elabbasy et al obtained Fibonacci sequence in solutions of some special cases of the following difference equation

$$
x_{n+1}=\frac{a x_{n-l} x_{n-k}}{b x_{n-p}-c x_{n-q}} .
$$

In [6], author deals with the behavior of the solution of the following nonlinear difference equation

$$
x_{n+1}=a x_{n-1}+\frac{b x_{n} x_{n-1}}{c x_{n}+d x_{n-2}} .
$$

Also, he gives specific forms of the solutions of four special cases of this equation. These specific forms also contain Fibonacci numbers. In [7], Cinar studied the positive solutions of the following difference equation system

$$
x_{n+1}=\frac{1}{y_{n}}, y_{n+1}=\frac{y_{n}}{y_{n-1} x_{n-1}} .
$$

In [10], Elsayed obtained the form of the solutions of the following rational difference system

$$
x_{n+1}=\frac{x_{n-1}}{ \pm 1+x_{n-1} y_{n}}, y_{n+1}=\frac{y_{n-1}}{\mp 1+y_{n-1} x_{n}} .
$$


In [12], Stevic examined the solutions of the following system of difference equations

$$
x_{n+1}=\frac{a x_{n-1}}{b y_{n} x_{n-1}+c}, y_{n+1}=\frac{\alpha y_{n-1}}{\beta x_{n} y_{n-1}+\gamma} .
$$

Now, we give information about Padovan numbers that establish a large part of our study. The Padovan sequence $\left\{P_{n}\right\}_{n \in \mathbb{N}}$, named after Richard Padovan, is defined by

$$
P_{n+1}=P_{n-1}+P_{n-2} \text {, with } P_{-2}=0, P_{-1}=0, P_{0}=1 .
$$

It can be easily obtained that the characteristic equation of (1.1) has the form

$$
x^{3}-x-1=0
$$

having the roots

$$
\left.\begin{array}{l}
p=\frac{r^{2}+12}{6 r} \\
p_{1}=-\frac{r^{2}+12}{6 r}-i \frac{\sqrt{3}}{2}\left(\frac{r}{6}-\frac{2}{3 r}\right) \\
p_{2}=-\frac{r^{2}+12}{6 r}+i \frac{\sqrt{3}}{2}\left(\frac{r}{6}-\frac{2}{3 r}\right)
\end{array}\right\},
$$

where $r=\sqrt[3]{108+12 \sqrt{69}}$. Furthermore, the unique real root $p$ is named as plastic number. Also there exists the following limit

$$
\lim _{k \rightarrow \infty} \frac{P_{k+1}}{P_{k}}=p,
$$

where $P_{k} \quad k$ th Padovan number. One can find more information associated with this sequence in $[16,17]$.

We will need the following definition in the sequel.

Definition 1.1 [18] Let $(\bar{x}, \bar{y})$ be an equilibrium point of a map $F=(f, g)$, where $f$ and $g$ are continuously differentiable functions at $(\bar{x}, \bar{y})$. The Jacobian matrix of $F$ at $(\bar{x}, \bar{y})$ is the matrix

$$
J_{F}(\bar{x}, \bar{y})=\left(\begin{array}{ll}
\frac{\partial f}{\partial x}(\bar{x}, \bar{y}) & \frac{\partial f}{\partial x}(\bar{x}, \bar{y}) \\
\frac{\partial g}{\partial x}(\bar{x}, \bar{y}) & \frac{\partial g}{\partial x}(\bar{x}, \bar{y})
\end{array}\right) .
$$

Also, suppose that $F=(f, g)$ is continuously differentiable on an open set $I$ in $\mathbb{R}^{2}$. Equilibrium point $(\bar{x}, \bar{y})$ is called a saddle point if one of the eigenvalues of $J_{F}(\bar{x}, \bar{y})$ is larger and another is less than 1 in absolute value.

In this study, we consider the solutions of the following two difference equation systems

$$
x_{n+1}=\frac{x_{n-1}+1}{y_{n} x_{n-1}}, y_{n+1}=\frac{y_{n-1}+1}{x_{n} y_{n-1}}
$$

and

$$
x_{n+1}=\frac{x_{n-1}-1}{y_{n} x_{n-1}}, y_{n+1}=\frac{y_{n-1}-1}{x_{n} y_{n-1}}
$$

such that their solutions are associated with Padovan numbers. We also establish a relationship between $\mathrm{Pa}-$ dovan numbers and the solutions of systems (1.3) and (1.4).

\section{Main Results}

In this section, we prove our main results. The following theorem studies the formulae of the solutions of systems (1.3) and (1.4) with initial conditions not making the denominator zero.

Teorem 2.1 Let $\left\{x_{n}, y_{n}\right\}_{n=0}^{\infty}$ denote the solutions of systems (1.3) and (1.4). Then, the forms of solutions $\left\{x_{n}, y_{n}\right\}_{n=0}^{\infty}$ are given by

$$
x_{n}= \begin{cases}\mp \frac{P_{n} x_{-1} y_{0} \mp P_{n+1} x_{-1}+P_{n-1}}{P_{n-1} x_{-1} y_{0} \mp P_{n} x_{-1}+P_{n-2}}, & \text { if } n \text { is odd } \\ \mp \frac{P_{n} y_{-1} x_{0} \mp P_{n+1} y_{-1}+P_{n-1}}{P_{n-1} y_{-1} x_{0} \mp P_{n} y_{-1}+P_{n-2}}, & \text { if } n \text { is even }\end{cases}
$$

and

$$
y_{n}=\left\{\begin{array}{ll}
\mp \frac{P_{n} y_{-1} x_{0} \mp P_{n+1} y_{-1}+P_{n-1}}{P_{n-1} y_{-1} x_{0} \mp P_{n} y_{-1}+P_{n-2}}, & \text { if } n \text { is odd } \\
\mp \frac{P_{n} x_{-1} y_{0} \mp P_{n+1} x_{-1}+P_{n-1}}{P_{n-1} x_{-1} y_{0} \mp P_{n} x_{-1}+P_{n-2}}, & \text { if } n \text { is even }
\end{array},\right.
$$

where $P_{n}$ be the $n$th Padovan number.

The following lemma is necessary for determining the initial conditions of the well-defined solutions of systems (1.3) and (1.4).

Lemma 2.2 (Forbidden Set) Forbidden sets of systems (1.3) and (1.4) are given by

$$
F_{1}=\bigcup_{n=-1}^{\infty}\left\{\left(x_{-1}, x_{0}, y_{-1}, y_{0}\right): A_{n}=0, B_{n}=0\right\},
$$

and

$$
F_{2}=\bigcup_{n=-1}^{\infty}\left\{\left(x_{-1}, x_{0}, y_{-1}, y_{0}\right): C_{n}=0, D_{n}=0\right\},
$$

where

$$
\begin{aligned}
& A_{n}=P_{n} y_{-1} x_{0}+P_{n+1} y_{-1}+P_{n-1}, \\
& B_{n}=P_{n} x_{-1} y_{0}+P_{n+1} x_{-1}+P_{n-1}, \\
& C_{n}=P_{n} y_{-1} x_{0}-P_{n+1} y_{-1}+P_{n-1}, \\
& D_{n}=P_{n} x_{-1} y_{0}-P_{n+1} x_{-1}+P_{n-1},
\end{aligned}
$$

respectively.

Proof of Theorem 2.1 We will just prove for system (1.3) since the other part can be proved in the same manner. We use the method of induction on $k$. For $k=0$, we have 


$$
\begin{aligned}
& x_{1}=\frac{x_{-1}+1}{y_{0} x_{-1}}=\frac{P_{1} x_{-1} y_{0}+P_{2} x_{-1}+P_{0}}{P_{0} x_{-1} y_{0}+P_{1} x_{-1}+P_{-1}} \\
& y_{1}=\frac{y_{-1}+1}{x_{0} y_{-1}}=\frac{P_{1} y_{-1} x_{0}+P_{2} y_{-1}+P_{0}}{P_{0} y_{-1} x_{0}+P_{1} y_{-1}+P_{-1}}
\end{aligned}
$$

For $k=1$, we obtain

$$
\begin{aligned}
& x_{2}=\frac{x_{0}+1}{y_{1} x_{0}}=\frac{x_{0}+1}{x_{0} \frac{y_{-1}+1}{x_{0} y_{-1}}}=\frac{P_{2} y_{-1} x_{0}+P_{3} y_{-1}+P_{1}}{P_{1} y_{-1} x_{0}+P_{2} y_{-1}+P_{0}} \\
& y_{2}=\frac{y_{0}+1}{x_{1} y_{0}}=\frac{y_{0}+1}{y_{0} \frac{x_{-1}+1}{y_{0} x_{-1}}}=\frac{P_{2} x_{-1} y_{0}+P_{3} x_{-1}+P_{1}}{P_{1} x_{-1} y_{0}+P_{2} x_{-1}+P_{0}} .
\end{aligned}
$$

Now, suppose that our assumption holds for $2 k-1$. That is;

$$
\begin{aligned}
& x_{2 k-2}=\frac{P_{2 k-2} y_{-1} x_{0}+P_{2 k-1} y_{-1}+P_{2 k-3}}{P_{2 k-3} y_{-1} x_{0}+P_{2 k-2} y_{-1}+P_{2 k-4}}, \\
& x_{2 k-1}=\frac{P_{2 k-1} X_{-1} y_{0}+P_{2 k} X_{-1}+P_{2 k-2}}{P_{2 k-2} X_{-1} y_{0}+P_{2 k-1} X_{-1}+P_{2 k-3}}, \\
& y_{2 k-2}=\frac{P_{2 k-2} X_{-1} y_{0}+P_{2 k-1} X_{-1}+P_{2 k-3}}{P_{2 k-3} X_{-1} y_{0}+P_{2 k-2} X_{-1}+P_{2 k-4}}, \\
& y_{2 k-1}=\frac{P_{2 k-1} y_{-1} x_{0}+P_{2 k} y_{-1}+P_{2 k-2}}{P_{2 k-2} y_{-1} x_{0}+P_{2 k-1} y_{-1}+P_{2 k-3}},
\end{aligned}
$$

From Equation (1.3), we can write for $2 k$,

$$
\begin{aligned}
x_{2 k}= & \frac{x_{2 k-2}+1}{y_{2 k-1} x_{2 k-2}}=\frac{\frac{P_{2 k-2} y_{-1} x_{0}+P_{2 k-1} y_{-1}+P_{2 k-3}}{P_{2 k-3} y_{-1} x_{0}+P_{2 k-2} y_{-1}+P_{2 k-4}}+1}{\left(\frac{P_{2 k-1} y_{-1} x_{0}+P_{2 k} y_{-1}+P_{2 k-2}}{P_{2 k-2} y_{-1} x_{0}+P_{2 k-1} y_{-1}+P_{2 k-3}}\right)\left(\frac{P_{2 k-2} y_{-1} x_{0}+P_{2 k-1} y_{-1}+P_{2 k-3}}{P_{2 k-3} y_{-1} x_{0}+P_{2 k-2} y_{-1}+P_{2 k-4}}\right)} \\
= & \frac{P_{2 k} y_{-1} x_{0}+P_{2 k+1} y_{-1}+P_{2 k-1}}{P_{2 k-1} y_{-1} x_{0}+P_{2 k} y_{-1}+P_{2 k-2}}
\end{aligned}
$$

and

$$
\begin{aligned}
y_{2 k}= & \frac{y_{2 k-2}+1}{X_{2 k-1} y_{2 k-2}}=\frac{\frac{P_{2 k-2} X_{-1} y_{0}+P_{2 k-1} X_{-1}+P_{2 k-3}}{P_{2 k-3} X_{-1} y_{0}+P_{2 k-2} X_{-1}+P_{2 k-4}}+1}{\left(\frac{P_{2 k-1} X_{-1} y_{0}+P_{2 k} X_{-1}+P_{2 k-2}}{P_{2 k-2} X_{-1} y_{0}+P_{2 k-1} X_{-1}+P_{2 k-3}}\right)\left(\frac{P_{2 k-2} X_{-1} y_{0}+P_{2 k-1} X_{-1}+P_{2 k-3}}{P_{2 k-3} X_{-1} y_{0}+P_{2 k-2} X_{-1}+P_{2 k-4}}\right)} \\
= & \frac{P_{2 k} X_{-1} y_{0}+P_{2 k+1} X_{-1}+P_{2 k-1}}{P_{2 k-1} X_{-1} y_{0}+P_{2 k} X_{-1}+P_{2 k-2}} .
\end{aligned}
$$

Similarly, from Equation (1.3), we obtain for $2 k+1$,

$$
\begin{aligned}
x_{2 k+1}= & \frac{x_{2 k-1}+1}{y_{2 k} X_{2 k-1}}=\frac{\frac{P_{2 k-1} X_{-1} y_{0}+P_{2 k} X_{-1}+P_{2 k-2}}{P_{2 k-2} X_{-1} y_{0}+P_{2 k-1} X_{-1}+P_{2 k-3}}+1}{\left(\frac{P_{2 k} X_{-1} y_{0}+P_{2 k+1} X_{-1}+P_{2 k-1}}{P_{2 k-1} X_{-1} y_{0}+P_{2 k} X_{-1}+P_{2 k-2}}\right)\left(\frac{P_{2 k-1} X_{-1} y_{0}+P_{2 k} X_{-1}+P_{2 k-2}}{P_{2 k-2} X_{-1} y_{0}+P_{2 k-1} X_{-1}+P_{2 k-3}}\right)} \\
= & \frac{P_{2 k+1} X_{-1} y_{0}+P_{2 k+2} X_{-1}+P_{2 k}}{P_{2 k} X_{-1} y_{0}+P_{2 k+1} X_{-1}+P_{2 k-1}}
\end{aligned}
$$

and

$$
\begin{aligned}
y_{2 k+1}= & \frac{y_{2 k-1}+1}{x_{2 k} y_{2 k-1}}=\frac{\frac{P_{2 k-1} y_{-1} x_{0}+P_{2 k} y_{-1}+P_{2 k-2}}{P_{2 k-2} y_{-1} x_{0}+P_{2 k-1} y_{-1}+P_{2 k-3}}+1}{\left(\frac{P_{2 k} y_{-1} x_{0}+P_{2 k+1} y_{-1}+P_{2 k-1}}{P_{2 k-1} y_{-1} x_{0}+P_{2 k} y_{-1}+P_{2 k-2}}\right)\left(\frac{P_{2 k-1} y_{-1} x_{0}+P_{2 k} y_{-1}+P_{2 k-2}}{P_{2 k-2} y_{-1} x_{0}+P_{2 k-1} y_{-1}+P_{2 k-3}}\right)} \\
= & \frac{P_{2 k+1} y_{-1} x_{0}+P_{2 k+2} y_{-1}+P_{2 k}}{P_{2 k} y_{-1} x_{0}+P_{2 k+1} y_{-1}+P_{2 k-1}},
\end{aligned}
$$


which completes the proof $\mathbf{m}$.

Theorem 2.3 The following statements hold:

1) System (1.3) has unique real equilibrium point $(p, p)$ and $(p, p)$ is a saddle point,

2) System (1.4) has unique real equilibrium point $(-p,-p)$ and $(-p,-p)$ is a saddle point, where $p$ is the plastic number.

\section{Proof}

1) Equilibrium point $(\bar{x}, \bar{y})$ of system (1.3) satisfy the system of equations

$$
\bar{x}=\frac{\bar{x}+1}{\bar{x} \cdot \bar{y}}, \bar{y}=\frac{\bar{y}+1}{\bar{x} \cdot \bar{y}} .
$$

In (2.3), by subtracting the second equation from the first equation and after some operations, we have

$$
(\bar{x} \cdot \bar{y}-1)(\bar{x}-\bar{y})=0 .
$$

For $\bar{x} \cdot \bar{y}=1$, the equations of (2.3) cannot be satisfied and so $\bar{x}=\bar{y}$. Consequently, we obtain the following cubic equation

$$
\bar{x}^{3}-\bar{x}-1=0 .
$$

The above cubic equation is the characteristic equation of the recurrence relation of the Padovan numbers in (1.2) having the unique real root $p$. Hence the unique equilibrium point of system (1.3) is point $(p, p)$. Now, we show that the equilibrium point is a saddle point. Firstly, system (1.3) is a special case of the general system of the form

$$
\begin{aligned}
& x_{n+1}=f\left(x_{n-1}, y_{n}\right), \\
& y_{n+1}=g\left(x_{n}, y_{n-1}\right),
\end{aligned}
$$

where $f(x, y)=\frac{x+1}{x y}$ and $g(x, y)=\frac{y+1}{x y}$. Then, we calculate the Jacobian of the corresponding map

$$
F(x, y)=(f(x, y), g(x, y)) .
$$

We get

$$
J_{F}(p, p)=\left(\begin{array}{cc}
-\frac{1}{p^{3}} & -\frac{p+1}{p^{3}} \\
-\frac{p+1}{p^{3}} & -\frac{1}{p^{3}}
\end{array}\right) .
$$

By taking into consideration (1.2), we obtain the characteristic equation of the Jacobian Matrix $J_{F}(p, p)$ as

$$
\left(\lambda+\frac{1}{p^{3}}\right)^{2}-1=0 .
$$

Hence, it is clearly seen that $\lambda_{1}=\left|1-\frac{1}{p^{3}}\right|<1$ and $\lambda_{2}=\left|-1-\frac{1}{p^{3}}\right|>1$, as desired

2) It can be proved in a similar manner.

Theorem 2.4 Let the initial conditions of the systems (1.3) and (1.4) be $x_{-1}, x_{0}, y_{-1}, y_{0} \notin F_{1}$ and $x_{-1}, x_{0}, y_{-1}, y_{0} \notin F_{2}$, respectively. Then the following statements hold:

1) The every solution of the system (1.3) converges to point $(p, p)$.

2) The every solution of the system (1.4) converges to point $(-p,-p)$.

Proof We will only prove for even-subscripted terms of $x_{n}$. Since the other parts of the proof are quite similar, they will be omited.

1) Let us take $n=2 k$ in (2.1). Then, we can write

$$
\begin{aligned}
x_{2 k} & =\frac{P_{2 k} y_{-1} x_{0}+P_{2 k+1} y_{-1}+P_{2 k-1}}{P_{2 k-1} y_{-1} x_{0}+P_{2 k} y_{-1}+P_{2 k-2}} \\
& =\frac{P_{2 k+1}}{P_{2 k}}\left(\frac{\frac{P_{2 k}}{P_{2 k+1}} y_{-1} x_{0}+y_{-1}+\frac{P_{2 k-1}}{P_{2 k+1}}}{\frac{P_{2 k-1}}{P_{2 k}} y_{-1} x_{0}+y_{-1}+\frac{P_{2 k-2}}{P_{2 k}}}\right) .
\end{aligned}
$$

Also, by taking into account $\lim _{k \rightarrow \infty} \frac{P_{k+1}}{P_{k}}=p$, we obtain the following equality

$$
\lim _{k \rightarrow \infty} x_{2 k}=p\left(\frac{\frac{1}{p} y_{-1} x_{0}+y_{-1}+\frac{1}{p^{2}}}{\frac{1}{p} y_{-1} x_{0}+y_{-1}+\frac{1}{p^{2}}}\right)=p,
$$

as desired

\section{Numerical Examples}

In order to illustrate and support theoretical results of the previous section, we consider several examples in this section. These examples represent the qualitative behavior of solutions of the mentioned nonlinear difference equation systems.

Example 3.1 Consider system (1.3) with the inital conditions $\quad x_{-1}=1.5, x_{0}=3.2, y_{-1}=1.9, y_{0}=2.3 \quad$ (See Figure 1).

Example 3.2 Consider system (1.4) with the inital conditions $\quad x_{-1}=2.5, x_{0}=1.2, y_{-1}=0.7, y_{0}=1.5 \quad$ (See Figure 2).

\section{Conclusion}

In this study, we formulated the solutions of equation systems (1.3) and (1.4) and determined their forbidden sets. Obtained formulae are given by means of Padovan numbers. Also, for $x_{-1}, x_{0}, y_{-1}, y_{0} \notin F_{1}$ and $x_{-1}, x_{0}, y_{-1}, y_{0} \notin F_{2}$, all the solutions of (1.3) and (1.4) interestingly tend to 


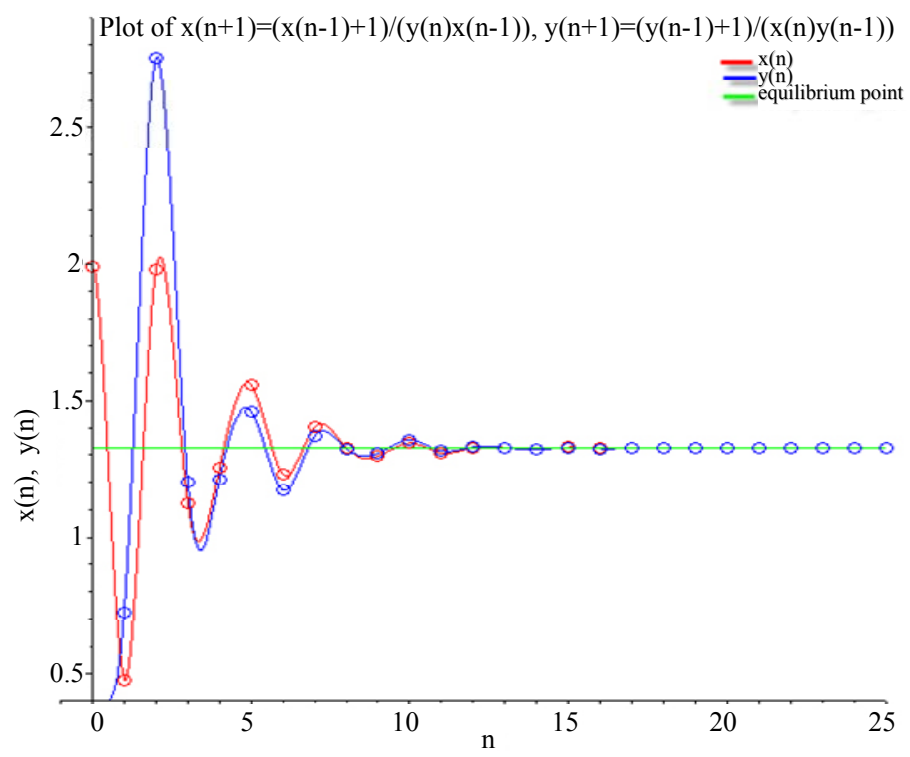

Figure 1. Plot of $x(n+1)=(x(n-1)-1) / y(n) x(n-1), y(n+1)=(y(n-1)-1) / x(n) y(n-1)$.

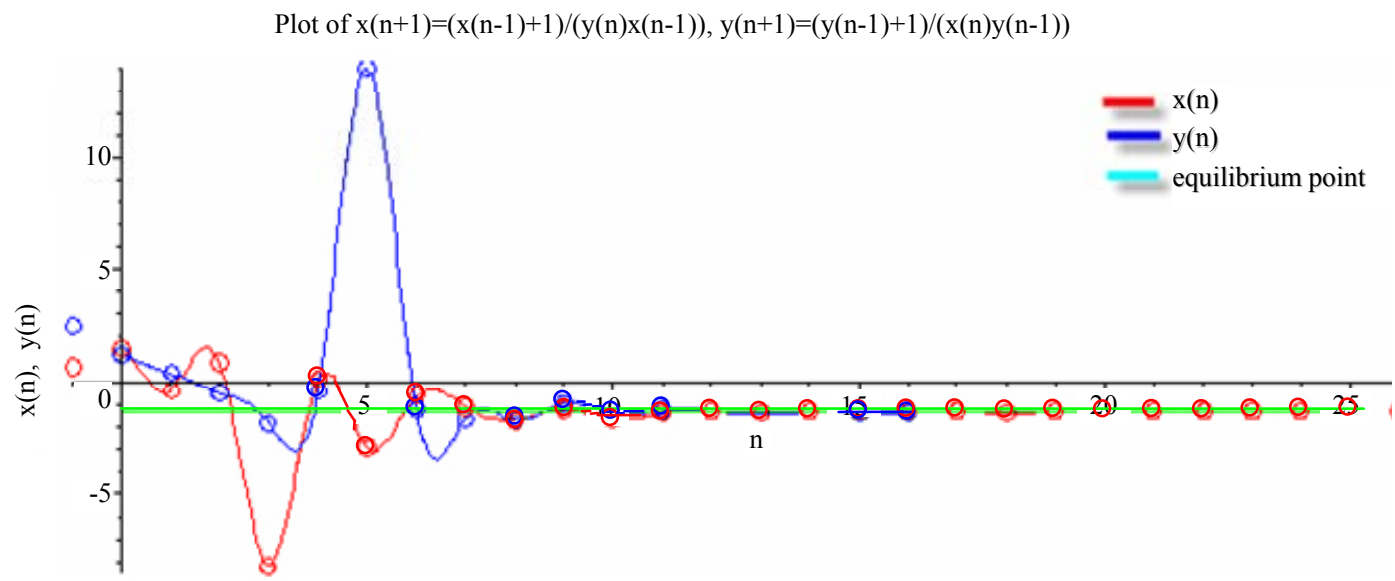

Figure 2. Plot of $x(n+1)=(x(n-1)-1) / y(n) x(n-1), y(n+1)=(y(n-1)-1) / x(n) y(n-1)$.

their equilibrium points $(p, p)$ and $(-p,-p)$, respectively, where $p$ is the plastic number.

\section{REFERENCES}

[1] R. P. Agarwal, "Difference Equations and Inequalities," Marcel Dekker, New York, 2000

[2] M. Aloqeili, "Dynamics of a Rational Difference Equation," Applied Mathematics and Computation, Vol. 176, No. 2, 2006, pp. 768-774. http://dx.doi.org/10.1016/j.amc.2005.10.024

[3] T. F. Ibrahim, "On the Third Order Rational Difference equation $x_{n+1}=\frac{x_{n} x_{n-2}}{x_{n-1}\left(a+b x_{n} x_{n-2}\right)}$," International Journal of Contemporary Mathematical Sciences, Vol. 4, No. 25-28, 2009, pp. 1321-1334.

[4] R. Khalaf-Allah, "Asymptotic Behaviour and Periodic
Naturel of Two Difference Equations," Ukrainian Mathematical Journal, Vol. 61, No. 6, 2009, pp. 988-993. http://dx.doi.org/10.1007/s11253-009-0249-2

[5] E. M. Elabbasy, H. A. El-Metwally and E. M. Elsayed, "Global Behavior of the Solutions of Some Difference Equations," Advances in Difference Equations, Vol. 2011, 2011, p. 28. http://dx.doi.org/10.1186/1687-1847-2011-28.

[6] E. M. Elsayed, "Solution and Attractivity for a Rational Recursive Sequence," Discrete Dynamics in Nature and Society, Vol. 2011, 2011, Article ID: 982309.

[7] C. Cinar, "On the Positive Solutions of the Difference Equation System $x_{n+1}=\frac{1}{y_{n}}, y_{n+1}=\frac{y_{n}}{y_{n-1} x_{n-1}}$," Applied Mathematics and Computation, Vol. 158, No. 2, 2004, pp. 303305. http://dx.doi.org/10.1016/j.amc.2003.08.073

[8] X. Yang, Y. Liu and S. Bai, "On the System of High Or- 
der Rational Difference Equations $x_{n}=\frac{a}{y_{n-p}}, y_{n}=\frac{b y_{n-p}}{x_{n-q} y_{n-q}}, "$ Applied Mathematics and Computation, Vol. 171, No. 2, 2005, pp. 853-856.

http://dx.doi.org/10.1016/j.amc.2005.01.092

[9] A. S. Kurbanli, C. Cinar and I. Yalcinkaya, "On the Behavior of Positive Solutions of the System of Rational Difference Equations," Mathematical and Computer Modelling, Vol. 53, No.5-6, 2011, pp. 1261-1267. http://dx.doi.org/10.1016/j.mcm.2010.12.009

[10] E. M. Elsayed, "Solutions of Rational Difference Systems of Order Two," Mathematical and Computer Modelling, Vol. 55, No. 3-4, 2012, pp. 378-384. http://dx.doi.org/10.1016/j.mcm.2011.08.012

[11] M. Mansour, M. M. El-Dessoky and E. M. Elsayed, "The Form of the Solutions and Periodicity of Some Systems of Difference Equations," Discrete Dynamics in Nature and Society, Vol. 2012, 2012, Article ID: 406821.

[12] S. Stevic, "On a System of Difference Equations," Applied Mathematics and Computation Vol. 218, No. 7, 2011, pp. 3372-3378. http://dx.doi.org/10.1016/j.amc.2011.08.079

[13] S. Stevic, "On Some Solvable Systems of Difference Equations," Applied Mathematics and Computation, Vol. 218, No. 9, 2012, pp. 5010-5018. http://dx.doi.org/10.1016/j.amc.2011.10.068

[14] D. T. Tollu, Y. Yazlik and N. Taskara, "On the Solutions of Two Special Types of Riccati Difference Equation via Fibonacci Numbers," Advances in Difference Equations, Vol. 2013, 2013, p. 174. http://dx.doi.org/10.1186/1687-1847-2013-174

[15] A. S. Kurbanli, C. Cinar and D. Simsek, "On the Periodicity of Solutions of the System of Rational Difference Equations $x_{n+1}=\frac{x_{n-1}+y_{n}}{y_{n} x_{n-1}-1}, y_{n+1}=\frac{y_{n-1}+x_{n}}{x_{n} y_{n-1}-1}$," Applied Mathematics, Vol. 2, No. 4, 2011, pp. 410-413. http://dx.doi.org/10.4236/am.2011.24050

[16] A. G. Shannon, P. G. Anderson and A. F. Horadam, "Properties of Cordonnier, Perrin and Van der Laan Numbers," International Journal of Mathematical Education in Science and Technology, Vol. 37, No. 7, 2006, pp. 825-831. http://dx.doi.org/10.1080/00207390600712554

[17] Benjamin M. M. De Weger, "Padua and Pisa are Exponentially Far Apart," Publicacions Matematiques, Vol. 41, No. 2, 1997, pp. 631-651. http://dx.doi.org/10.5565/PUBLMAT 41297 23

[18] M. R. S. Kulenovic and O. Merino, "Discrete Dynamical Systems and Difference Equations with Mathematica," A CRC Press Company, NewYork, 2002. 